\title{
Evaluación de la Cadena de Suministro para Mejorar la Competitividad y Productividad en el Sector Metalmecánico en Barranquilla, Colombia
}

\author{
Katherinne Salas-Navarro ${ }^{1,2^{\star}}$, Jhadai A. Meza ${ }^{1}$, Thalía Obredor-Baldovino ${ }^{1}$ y Nohora Mercado-Caruso ${ }^{1}$ \\ (1) Departamento de Gestión Industrial, Agroindustria y Operaciones, Universidad de la Costa, \\ Calle 58 No. 55-66, Barranquilla-Colombia. (e-mail: ksalas2@cuc.edu.co) \\ (2) Facultad de Ingeniería, Universidad Pontificia Bolivariana, Circ. 1 N 73-34, Medellín, Colombia.
}

${ }^{*}$ Autor a quien debe ser dirigida la correspondencia.

Recibido Jun. 20, 2018; Aceptado Ago. 8, 2018; Versión final Nov. 19, 2018, Publicado Abr. 2019

\begin{abstract}
Resumen
El presente artículo estudia los niveles de competitividad y productividad del Sector Metalmecánico de la ciudad de Barranquilla, a partir del diseño de un modelo de evaluación para la cadena de suministro de empresas pertenecientes a dicho sector. El modelo está conformado por cinco aspectos críticos: abastecimiento, almacenamiento, gestión de inventarios, distribución y transporte y logística inversa. Estos factores incluyen un conjunto de variables que evalúan la planeación, ejecución, medición y control de la gestión de la cadena de suministro. El modelo fue validado en cinco empresas del Sector Metalmecánico de la ciudad de Barranquilla. Se establecieron soluciones para las prácticas de logística integral en los procesos de aprovisionamiento, teniendo en cuenta normas de calidad de procesos y seguridad de la cadena de suministro. Se concluye que el sector ha presentado un gran crecimiento económico en el país y se hace necesario la optimización y mejora continua de los procesos productivos a través del monitoreo y control de indicadores apropiados.
\end{abstract}

Palabras clave: sector metalmecánico; competitividad; productividad; cadena de suministro; modelo de evaluación

\section{Evaluation of the Supply Chain to Improve Competitiveness and Productivity in the Metalworking Industry in Barranquilla, Colombia}

\begin{abstract}
This paper studies the levels of competitiveness and productivity of the Metalworking Sector of the city of Barranquilla, based on the design of an evaluation model for the supply chain of companies belonging to that sector. The model consists of five critical aspects: supply, storage, inventory management, distribution and transport, and reverse logistics. These factors include a set of variables that evaluate the planning, execution, measurement, and control of supply chain management. The model was validated in five companies of the Metalworking Sector of the city of Barranquilla. Solutions for integral logistics practices were established in the procurement processes, taking into account quality standards of processes and security of the supply chain. It is concluded that the sector has presented a great economic growth in the country and it is necessary the optimization and continuous improvement of the productive processes through the monitoring and control of appropriate indicators.
\end{abstract}

Keywords: metalworking industry; competitivity; productivity; supply chain; evaluation model 


\section{INTRODUCCIÓN}

Actualmente el sector metalmecánico se ha convertido en una fuente de ingreso en crecimiento en el país de Colombia, generando empleos formales e informales y el desarrollo de proyectos, colaborativos gubernamentales. La industria metalmecánica y la persistente competitividad de los mercados permite que las empresas diariamente se esfuercen por desarrollar con mayor eficiencia no solo en las labores productivas sino también las operaciones logísticas de aprovisionamiento y distribución. La cadena de suministro representa entonces una red de suministro de material, su transformación en un producto terminado y la distribución de estos productos a los clientes finales generando calidad en los procesos (Salas-Navarro, Acevedo-Chedid, Mercado-Caruso, y Sana, 2018; Salas-Navarro, Maiguel-Mejia, y Acevedo-Chedid, 2017). La cadena de suministro incluye capacidad de respuesta, eficiencia y control (Kathawalay Abdou, 2003). Para aumentar la ventaja competitiva, muchas empresas consideran que un sistema de cadena de suministro bien diseñado e implementado es una herramienta importante (Chen et al., 2006).

Las empresas buscan y venden en el mercado global buscando nuevos proveedores, socios, canales de distribución y también nuevas posibilidades de crecimiento y rentabilidad (Viana, 2014). La creciente competitividad y la globalización exigen respuestas eficientes por parte de las empresas y estrategias que les permitan sobrevivir en un mundo en continuo cambio (Salas Navarro, 2011) y a reducir los tiempos de espera por parte de los clientes (Nuñez et al., 2017). Bernal et al. (2012) plantean que cada negocio depende de otros negocios en su propio campo, lo que hace que las empresas deban coordinar esfuerzos para alcanzar mayores niveles de competitividad, y también afirman que la gestión de cadena de abastecimiento, o supply chain management, como coordinación estratégica y sistemática de las funciones convencionales del negocio, dentro de la compañía y entre la compañía y su cadena de abastecimiento, permiten mejorar el desempeño de la misma a largo plazo, teniendo en cuenta que de acuerdo a Lambert y Stock (2007) el resultado de un conjunto de actividades es más que la suma de cada actividad que la conforma. Aldana-Bernal y BernalTorres (2018) estudiaron los factores blandos como capital social, cultura organizacional y gestión del conocimiento en la gestión de cadenas de suministro como estrategia de fortalecimiento de la competitividad de las empresas.

Además, Peñafiel (2016) menciona que es necesario antes de planear el diseño de un plan estratégico corporativo o de logística, es necesario tener claridad del contexto en el que se va a desarrollar, por lo que es importante entonces definir un diagnóstico, metas y recursos necesarios. Sumado a que actualmente, para muchas empresas es necesario la estandarización de sus procesos y procedimientos como requisito y apoyo en la implementación de un sistema de gestión de calidad que permita, por medio del mismo, obtener una estructura eficiente y competitiva, que haga que la empresa sobre salga entre sus competidores y brinde mayor satisfacción a sus clientes (Ospina-Mateus et al., 2017). A su vez, Kara et al. (2007) manifiesta que, dado los intereses de los consumidores, la responsabilidad de las empresas va más allá de la producción y distribución de sus productos. La competencia en el entorno empresarial global ha obligado a las empresas a ver toda la cadena de suministro de una manera integradora (Ernst y Kamrad, 2000).

La gestión efectiva de las cadenas de suministro de una organización ha demostrado ser un mecanismo muy eficaz para proporcionar una entrega rápida y confiable de productos y servicios de alta calidad al menor costo. Para lograr esto, la evaluación del desempeño de toda la cadena de suministro es extremadamente importante (Liang, Yang y Cook, 2006). Las problemáticas de almacenamiento, de producción, de comercialización, de aprovisionamiento, de inventario, de distribución y transporte, de logística inversa, de logística verde, entre otras, son causales que generan gran incertidumbre y sobrecostos en las empresas, por ende, es necesaria la identificación de los factores que dificultan el óptimo desarrollo de las operaciones logísticas en las organizaciones. De la misma manera es importante el fortalecimiento de las capacidades y recursos de las empresas de este sector con el fin de que sean más dinámicas e innovadoras lo que se refleja en el crecimiento económico (Camargo Acuña y Diaz Alonso, 2017). Las organizaciones requieren estructuras apropiadas para permitir tanto la evaluación como la aplicación de medidas externas (Hervani, Helms y Sarkis, 2005). Una medida global de desempeño puede ser un criterio apropiado para una cadena de abastecimiento dentro de una organización; sin embargo, el desempeño local se convierte en una medida importante para cadenas de suministro interorganizacionales (Swaminathan, SmithySadeh, 1998).

Una adecuada alternativa es el diseño y desarrollo de una herramienta de evaluación que permita conocer a través de indicadores, el estado de los procesos productivos de las empresas con relación a toda su cadena de abastecimiento, debido a que el aumento del grado de integración de la cadena de suministro creará mayores beneficios potenciales obtenibles (Rosenzweig et al., 2003). Además de la integración dentro de la empresa, la gestión del suministro la cadena implica una conexión entre empresas y, por lo tanto, un marco de gestión de la cadena de suministro debe influir en la forma en que se gestionan estas interfaces (Lambert, García-Dastugue y Croxton, 2005).También, es importante identificar los factores determinantes de la conducta intraemprendedora que favorecen la autonomía en el trabajo, el desempeño del trabajador y 
fomentan la productividad dentro de la empresa (Cabana, Narea y Orrego; 2018). Todo este diseño se realizará a través del desarrollo de un estudio experimental junto con el análisis del diagnóstico previo de la cadena de suministro de cada una de las empresas a las cuales les sea aplicada dicha herramienta, implementando mejores soluciones a los problemas logísticos integrales de toda la cadena de suministros.

En Colombia se han realizado estudios relacionados con la gestión, productividad y competitividad del Sector Metalmecánico. Posada (2004) desarrolló un estudio en empresas del sector para evaluar el grado de implementación de las prácticas en manufactura exitosas a nivel mundial y conocer los factores críticos de éxito, a partir de la experiencia en las empresas. Trujillo y Iglesias (2012) estimaron el crecimiento de las micro, pequeñas y medianas empresas del Sector Metalmecánico durante 2002-2006, identificando como factores claves de crecimiento la competencia local y los costos laborales. Ovalle, Ocampo y Acevedo (2013) identificaron las brechas tecnológicas en automatización industrial de las empresas del sector metalmecánico de Caldas encontrando falencias en la gestión y planes tecnológicos. De igual forma, Hernández, Cardona, y Del Rio (2017) han establecido los niveles de innovación en tecnología y direccionamiento estratégico de pequeñas y medianas empresas en Barranquilla. Aunque se ha realizado un trabajo significativo sobre la medición y gestión del rendimiento en las operaciones internas de las empresas, el énfasis en la medición del rendimiento de la cadena de suministro (especialmente en el sector metalmecánico), ha sido relativamente limitado (Lima Junior y Cesar Ribeiro, 2017), por lo que se hace necesario analizar el comportamiento de la cadena de suministro en este sector, que hasta el momento han recibido poca atención.

Se propone una investigación con el objetivo de medir los niveles de competitividad y productividad del Sector Metalmecánico de la ciudad de Barranquilla (Colombia). Como primera medida se debe planificar y controlar el diseño y desarrollo de la herramienta, para lo cual se determina las etapas del diseño y desarrollo de la herramienta y la revisión, verificación y validación, apropiadas para cada etapa del diseño y desarrollo, y las pautas dadas en cada fase de la investigación. De igual forma, se deben gestionar las interfaces entre los diferentes paquetes de trabajo involucrados en el diseño y desarrollo para asegurarse de una comunicación eficaz y una clara asignación de responsabilidades. Los resultados de la planificación deben actualizarse, según sea apropiado, a medida que progresa la investigación.

\section{METODOLOGÍA}

La metodología presentada en este documento se centra en plantear soluciones para las prácticas de la logística integral en las empresas del sector metalmecánico que funcionan en la ciudad de Barranquilla, enfocándose en los procesos productivos de cada uno de los aspectos fundamentales de la cadena de aprovisionamiento, e identificando las fortalezas y debilidades que presentan con el fin de mejorarlas.

En primera instancia se determinaron los elementos de entrada para el diseño y desarrollo. Estos elementos de entrada incluyeron los requisitos funcionales y de desempeño, los requisitos legales y reglamentarios aplicables, y la información proveniente de diseños previos similares. Cumplidos los requisitos de entrada, se validó la herramienta mediante la participación de las empresas encuestadas asegurando la satisfacción de los requisitos previstos. La herramienta de evaluación fue implementada en el software Microsoft Excel (2013) en el que se incluyeron los aspectos a evaluar en la cadena de suministro: abastecimiento, almacenamiento, inventario, distribución y transporte y logística inversa. Cada aspecto está conformado por un conjunto de variables que evalúan todos los aspectos relacionados con la planeación de actividades, cumplimiento de las mismas, relaciones entre los diferentes actores de la cadena, definición de políticas, competencias y habilidades del personal, entre otros, que permiten evaluar en la totalidad cada aspecto de la cadena de abastecimiento. Y adicionalmente se dieron parámetros de evaluación para cada resultado.

Para la modelación de la herramienta se desarrolló una lluvia de ideas y preguntas acerca de cómo realizar una adecuada gestión de análisis en los procesos de la cadena logística de las empresas del sector metalmecánico. A cada pregunta se le estableció una ponderación a criterio del investigador principal. Cada aspecto estuvo conformado por una serie de variables que evalúan actividades relacionadas con la planeación el cumplimiento, las relaciones entre los diferentes actores de la cadena, la definición de políticas, competencias y habilidades del personal, entre otros. Y adicionalmente se dieron parámetros de evaluación para cada resultado que arroje cada aspecto en su posterior evaluación.

\section{PROPUESTA DE MODELO}

El modelo de evaluación de la cadena de suministro para mejorar la competitividad y productividad se basa en el Modelo de Bechmarking de la Cadena de Abastecimiento para Pymes Manufactureras, desarrollado por (Pérez-Olivera et al., 2010) y la implementación en empresas del PRODES (Programa de Desarrollo Sectorial) de alimentos de Acopi, de la ciudad de Cali. Para evaluar el estado de la estructura logística se tuvieron como base algunos lineamientos dados por Navarro (2013) y los procesos productivos de las cadenas de 
abastecimiento del Sector Metalmecánico de la ciudad de Barranquilla. A partir de éstos trabajos, se establecen los aspectos a evaluar en la cadena de abastecimiento, tales como abastecimiento, almacenamiento, inventario, distribución y transporte y logística Inversa, con el propósito de evaluar los niveles de competitividad y productividad de las empresas.

En la Figura 1 se presenta el diagrama del modelo de la cadena de abastecimiento, conformado por cinco aspectos claves: abastecimiento, almacenamiento, inventario, distribución y transporte y logística inversa, que interactúan entre sí en medio de la gestión organizacional en un proceso de mejora continua con actividades de planificación, ejecución, seguimiento y control. El modelo a nivel interno de la empresa mide la productividad o la eficiencia de los procesos y aprovechamiento de los recursos y a nivel externo mide la competitividad de la empresa en el medio empresarial.

Cabe resaltar que la herramienta propuesta tiene una gran contribución de aplicación debido a que predice el comportamiento de cada uno de los procesos dentro de la cadena de abastecimiento y permite conocer los factores críticos de la organización brindando estrategias para optimizar los procesos logísticos y/o productivos de la cadena de suministros en empresas del sector metalmecánico.

El abastecimiento busca consolidar la adquisición de materia prima e insumos necesarios para la transformación de estos en productos terminados. De igual forma, debe garantizar la calidad de los insumos, proveedores y del proceso en general para contribuir con la productividad, eficiencia de los procesos organizaciones y la competitividad. El almacenamiento es un proceso necesario para la gestión eficiente del flujo de materiales y productos en un almacén, manejo eficiente, manipulación, empaque y cumplimiento de tiempos. La gestión de inventarios es un aspecto clave muy relacionado con el almacenamiento que permite planificar, controlar y garantizar la disponibilidad de insumos, materia prima, equipos y productos de forma eficiente. La distribución y transporte es un aspecto clave que incluye la gestión de flujos desde un punto de origen hacia un punto de destino, optimizando los tiempos de entrega, diseño de rutas, selección de flota y transportadores y programación de despachos. Finalmente, la logística inversa es aspecto que permite retroalimentar la cadena de suministro con el cliente final, a través de la gestión eficiente de los flujos de productos desde el mercado hasta el punto de origen con el propósito de recuperar su valor, reaprovechamiento de recursos, devoluciones y disposición final. La logística inversa eleva el nivel de ventas y trae consigo la utilización eficiente de los recursos (menor costo) y protección ambiental (Abdulrahmanet al., 2014).

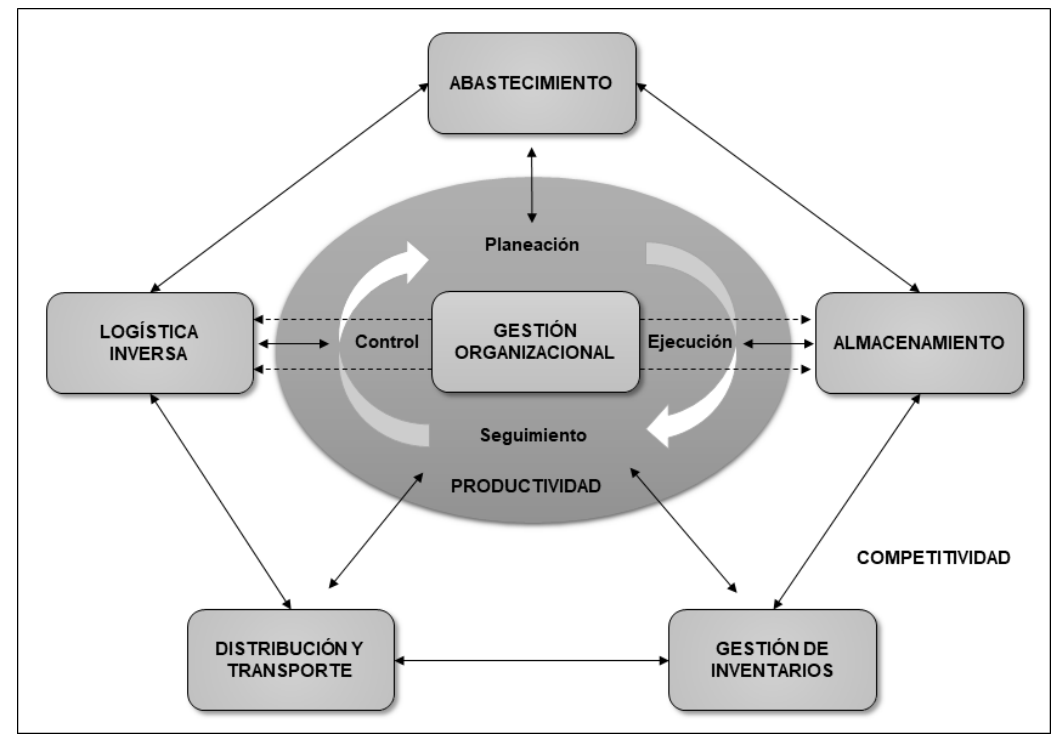

Fig. 1: Diagrama del modelo de la cadena de abastecimiento.

Cada aspecto está conformado por un conjunto de variables que evalúan todos los aspectos relacionados con la planeación de actividades, cumplimiento de las mismas, relaciones entre los diferentes actores de la cadena, definición de políticas, competencias y habilidades del personal, entre otros, que permiten evaluar en la totalidad cada aspecto de la cadena de abastecimiento. Adicionalmente se dieron parámetros de evaluación para cada resultado.

La figura 2 presenta la estructura de cada uno de los aspectos, los cuales están conformados por parámetros que establecen una escala de altamente, medianamente, poco y no confiable; módulos que incluyen procesos de planificación, ejecución y medición y control que están inmersos en un proceso de mejora continua que garantice la calidad, la productividad y competitividad de las empresas que conforman la cadena de suministro. 


\section{VALIDACIÓN DEL MODELO PROPUESTO}

La validación del modelo propuesto se realiza en cinco empresas líderes del Sector Metalmecánico de la ciudad Barranquilla, Colombia, teniendo en cuenta que solo éstas representan más de $35 \%$ del total de ventas del sector manufacturero (Revista Dinero, 2015).

A cada una de las empresas se le aplicó la herramienta de diagnóstico construida a partir del modelo de evaluación de la cadena de suministro para identificar el estado actual en materia de productividad y competitividad de acuerdo a los indicadores establecidos para cada aspecto a evaluar: abastecimiento, almacenamiento, gestión de inventarios, distribución y transporte y logística inversa.

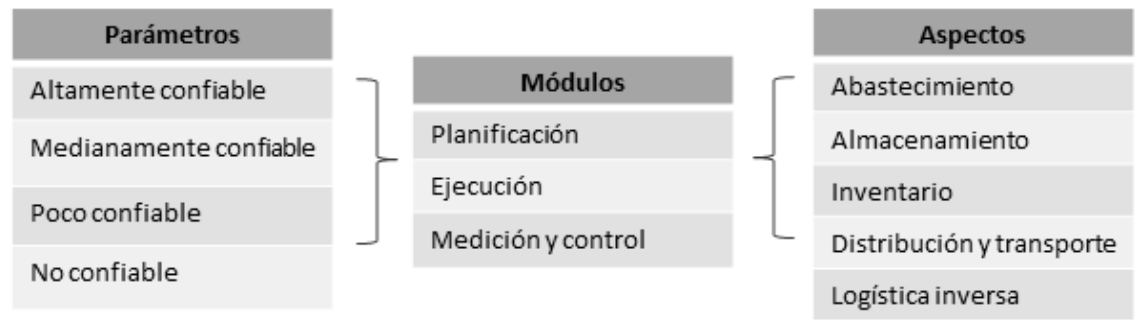

Fig. 2: Estructura de los Aspectos

En las figuras 3-7, se puede observar el panorama de las empresas con respecto a la planificación del proceso de compras y de adquisición de materiales e insumos necesarios para los procesos productivos. Solamente el $60 \%$ de las empresas encuestadas realiza la medición del cumplimiento de los proveedores teniendo en cuenta las entregas a tiempo y las devoluciones por no calidad, el porcentaje de empresas restante no realiza registro de las devoluciones por no calidad o por averías. Se evidencia la falta de uso de sistemas de información y software especializados para realizar los pedidos, solamente el $80 \%$ de las empresas realizan sus pedidos a través de EDI (Intercambio de datos electrónicos). El $60 \%$ de las empresas establece relaciones con sus proveedores para obtener materias primas a bajo costo, y solamente al $40 \%$ les interesa el establecimiento de asociaciones para realizar esfuerzos en conjunto y compartir información. El $20 \%$ de las empresas realiza un control de sus inventarios de forma manual.

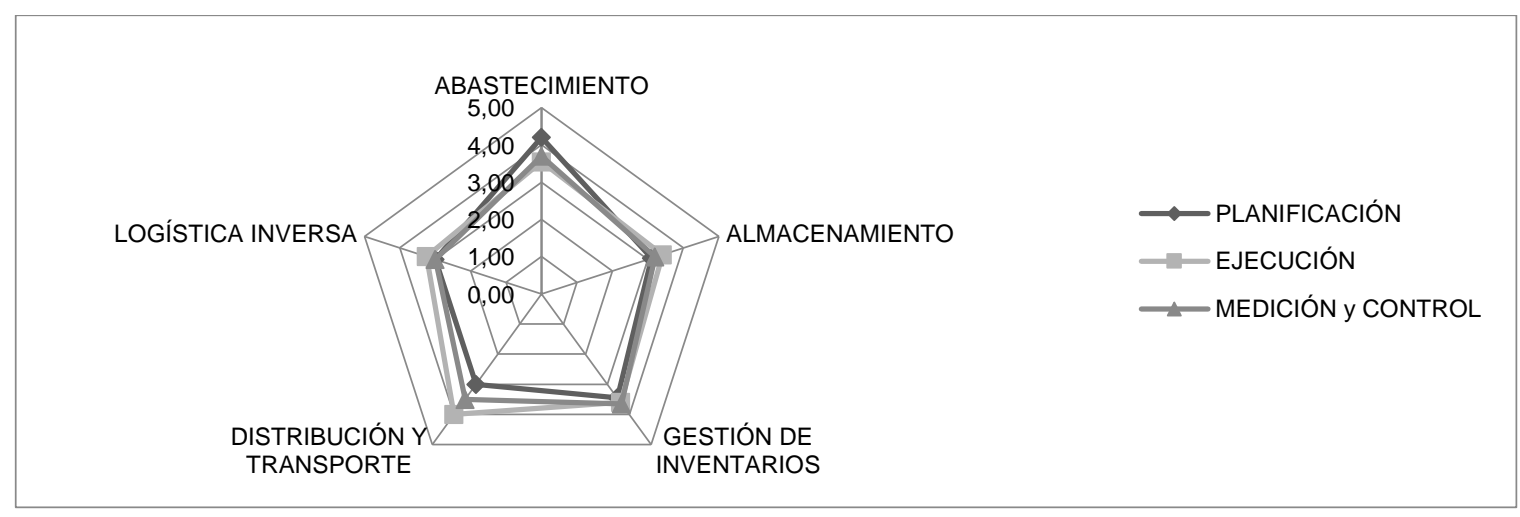

Fig. 3: Resultados de empresa 1.

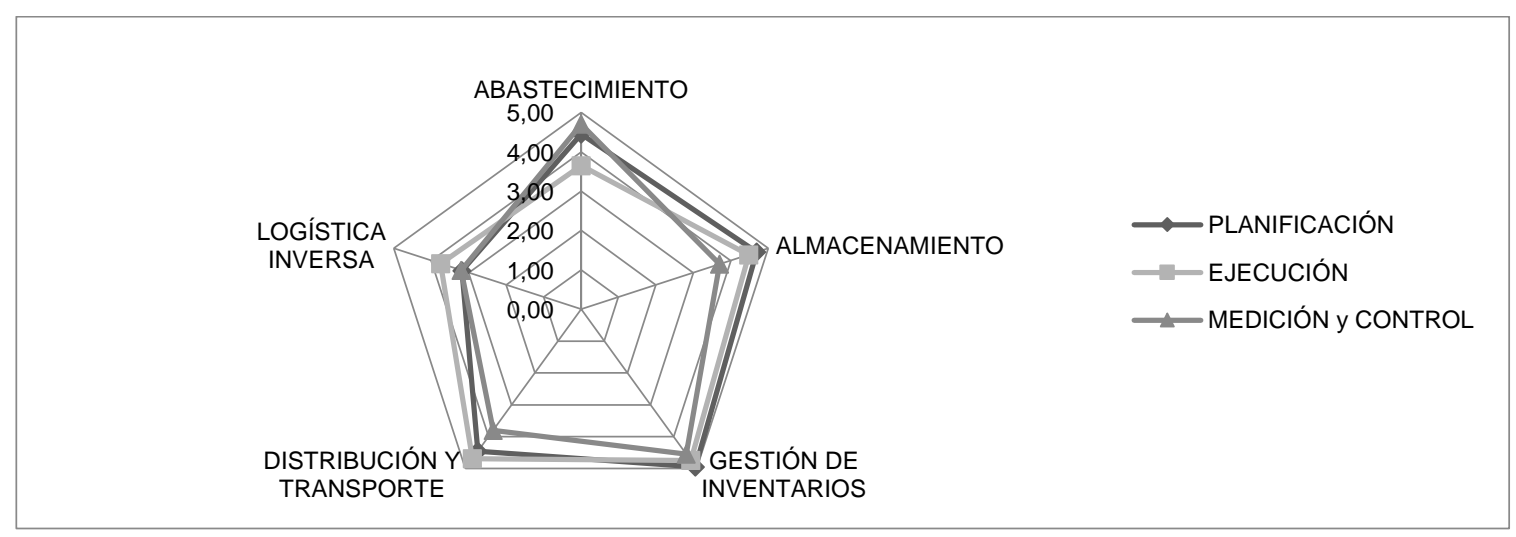

Fig. 4: Resultados de empresa 2. 


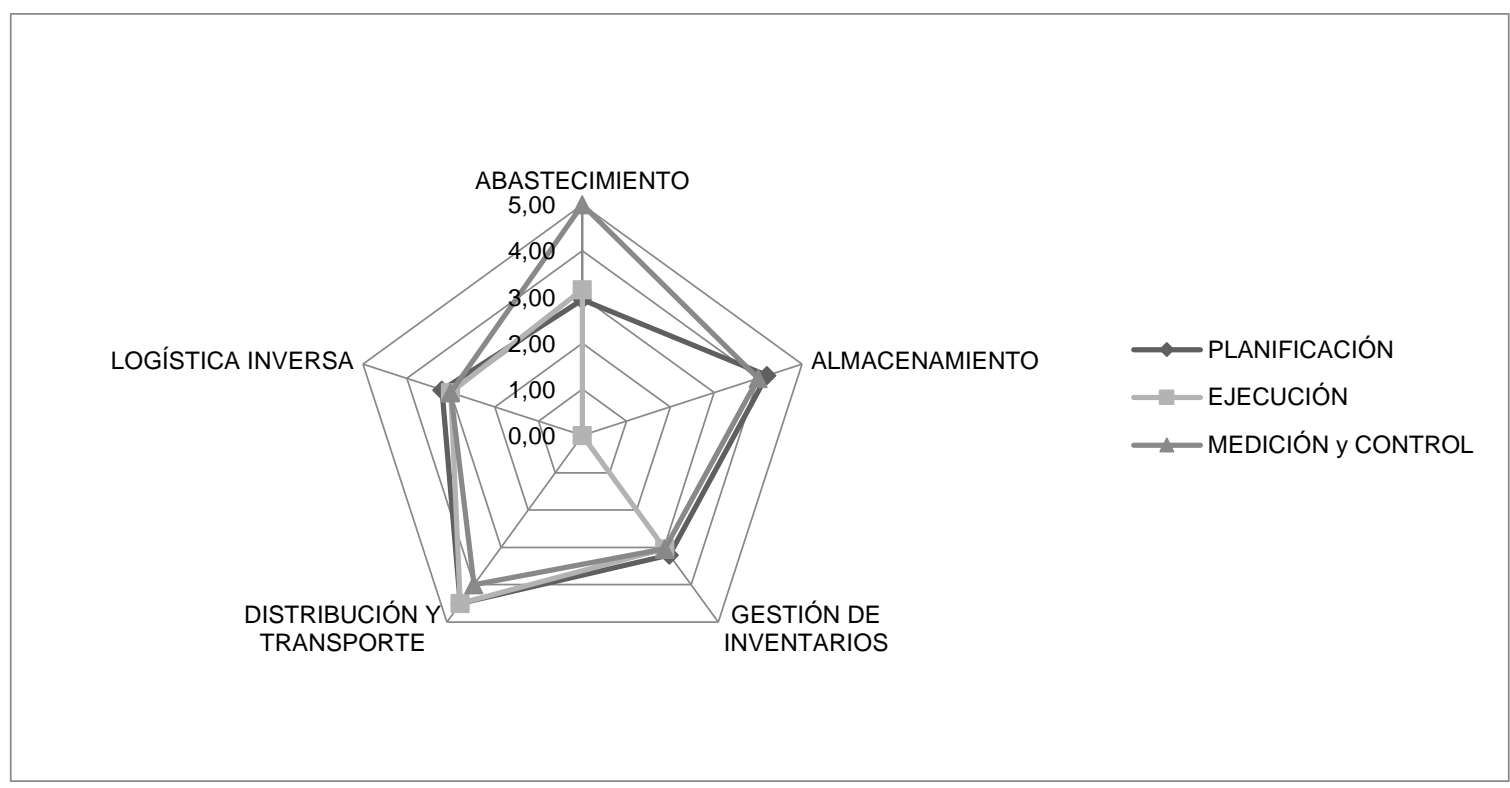

Fig. 5: Resultados de empresa 3

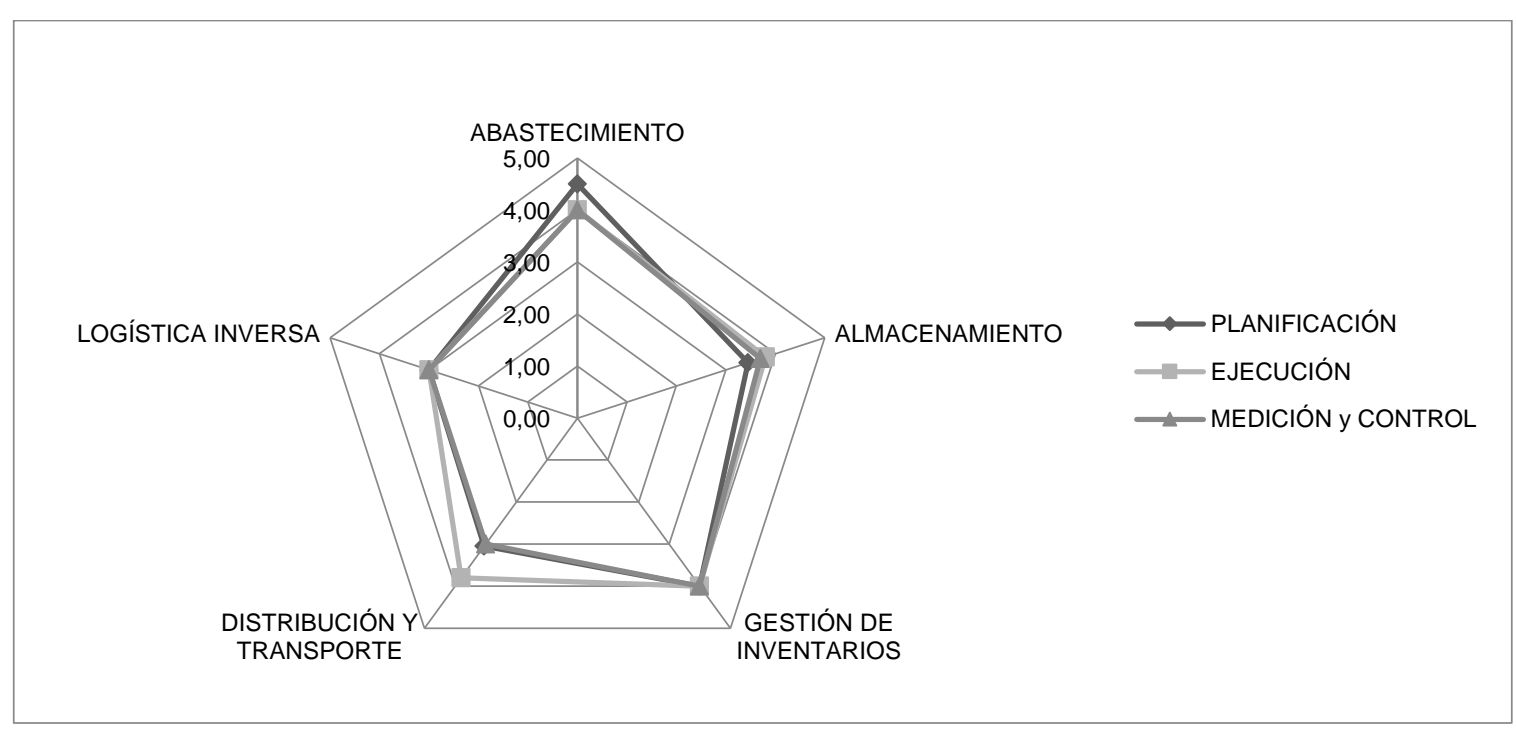

Fig. 6: Resultados de empresa 4

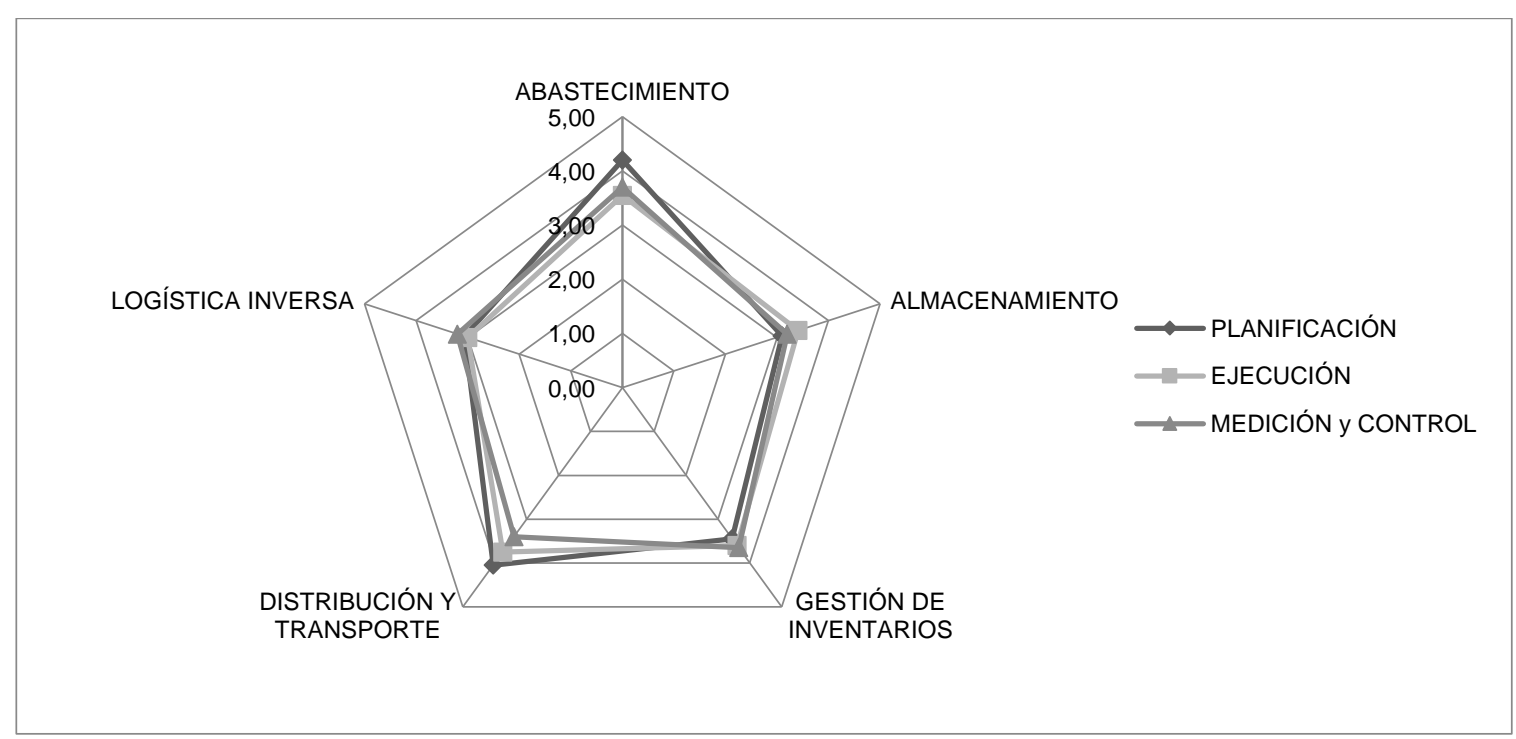

Fig. 7: Resultados de empresa 5. 
Las empresas han establecido niveles mínimos y máximos para mantener materia prima en inventario y para ordenar. El Almacenamiento es un proceso de planificación y control de las existencias físicas, para garantizar la disponibilidad de los productos (materias primas, partes, productos en proceso y producto terminado) de forma efectiva y a costos razonables, entre los puntos de origen y de llegada al cliente en cada proceso. Para garantizar el proceso de almacenamiento es necesario manejar los productos correctos en las unidades, correctas; manejo eficiente de los productos para evitar daños y averías, de tal forma que se entreguen en perfectas condiciones; establecer tiempos de preparación (selección y embalaje), para asegurar el cumplimiento en la entrega a tiempo del pedido; y, finalmente, diligenciar adecuadamente las órdenes de salidas y manejar una información veraz en los sistemas de información, para evitar errores de comunicación y documentación en la entrega de mercancías.

El panorama del sector metalmecánico en la ciudad de Barranquilla muestra los siguientes elementos: El $60 \%$ de las empresas cuenta con políticas de almacenamiento de materia prima y de producto terminado definidas. El $40 \%$ de las empresas realiza el inventario de materia prima y producto terminado de forma manual y el $60 \%$ lo realiza a través de software especializados, lo que evidencia una brecha tecnológica con respecto a otras empresas de talla mundial. En las empresas 1 y 3 , se realiza el manejo de materiales de forma manual, sin ayuda de equipos que faciliten su manipulación. Se evidencia que los partícipes en éste proceso, quienes realizan el control de entradas y salida de productos de materia prima y/o producto terminado, no es personal tecnificado y capacitado para tal fin.

La distribución constituye un conjunto de actividades que permite el flujo de salida de materiales y/o productos terminados desde la fábrica de producción hasta el cliente final. El transporte hace referencia a los medios o modos a través de los cuales se desarrolla la función de distribución dentro de una cadena de abastecimiento. El transporte incluye los traslados internos y externos de las materias primas, productos en proceso y productos terminados para su comercialización. De igual forma, debe garantizar los traslados de retorno de los productos y/o materiales que así se requieran. Al momento de seleccionar el modo de transporte, es necesario tener en cuenta criterios como el costo, los volúmenes que se deseen transportar, los tiempos de respuesta de la cadena de abastecimiento y las variaciones en el mismo, la flexibilidad y la entrega a tiempo de los productos a los usuarios finales. Se evidencia que el $80 \%$ de las empresas comercializa sus productos de forma directa y solo el $20 \%$ lo hace a través de intermediarios, lo que muestra que gran parte de las empresas distribuye directamente sus productos y los entrega a los clientes finales.

\section{CONCLUSIONES}

Se infiere que el modelo propuesto reproduce sistemas reales de producción, por consiguiente, es de gran utilidad para efectuar un estudio detallado del comportamiento de cada uno de los procesos dentro de la cadena de abastecimiento, la capacidad y tiempos que no se podrían calcular con certeza, de forma detallada como la herramienta lo plantea. De esta manera, se facilitan los procesos operacionales y se contribuye al crecimiento de las organizaciones, obteniendo certificaciones de calidad que son vistas de manera positiva por sus clientes y/o futuros clientes. Así mismo, se puede concluir que el Sector Metalmecánico ha presentado un gran crecimiento económico en el país, y por esta razón, la optimización y mejora continua de las empresas en sus operaciones es fundamental para un mejor desempeño de las mismas, obligando a que las empresas monitoreen, y controlen sus procesos productivos. Considerando esto, esta herramienta podría ser implementada en procesos de investigación de campo en empresas con el fin de establecer el estado actual de la empresa en la que se implemente.

\section{REFERENCIAS}

Abdulrahman, M.D., A. Gunasekaran y N. Subramanian, Critical Barriers In Implementing Reverse Logistics In The Chinese Manufacturing Sectors, International Journal of Production Economics, 147, 460-471(2014)

Aldana-Bernal, J.C. y C.A. Bernal-Torres, Factores Blandos en la Gestión de Integración de las Cadenas y/o Redes de Abastecimiento: Aproximación a un Modelo Conceptual, Información Tecnológica, 29(2), 103-114 (2018)

Bernal Torres, C.A., G. Fracica Naranjo y J.S. Frost Gonzalez, Análisis de la Relación entre la Innovación y La Gestión del Conocimiento con la Competitividad Empresarial en Una Muestra de Empresas en La Ciudad De Bogotá, Estudios Gerenciales, 28(spe), 303-315(2012)

Cabana, S.R., H.J. Narea y R.D. Orrego, Factores Determinantes de la Conducta Intraemprendedora en Pequeñas y Medianas Empresas (PyMes) de la Región Coquimbo en Chile, Información Tecnológica, 29(4), 167-178 (2018)

Chen-Tung C., L. Ching-Torng, C. Sue-Fn Huang, A Fuzzy Approach For Supplier Evaluation And Selection In Supply Chain Management. Int. J., Production Economics 102, 289-301(2006)

Ernst, R. y B. Kamrad, Evaluation of Supply Chain Structures through Modularization and Postponement, European Journal of Operational Research, 124, 495-510(2000) 
Hernández, H.G., D.A. Cardona y J.L. Del Rio, Direccionamiento Estratégico: Proyección de la Innovación Tecnológica y Gestión Administrativa en las Pequeñas Empresas, Información Tecnológica, 28(5), 15-22 (2017)

Hervani, A., M. Helms y J. Sarkis, Performance Evaluation of a Supply Chain Network, Benchmarking an International Journal, 12(4), 330-353 (2005)

Kara S., F. Rugrungruang y H. Kaebernick, Simulation Modelling of Reverse Logistics Networks, International Journal of Production Economics, 106, 61-69(2007)

Kathawala, Y. y K. Abdou, Supply Chain Evaluation in the Service Industry: A Framework Development Compared To Manufacturing, Managerial Auditing Journal, 18(2), 140-149 (2003)

Lambert, D., S. García-DastugueyK. Croxton, An Evaluation of Process-Oriented Supply Chain Management Frameworks, Journal of Business Logistics, 26(1), 25-51(2005)

Liang, L., F. Yangy, W. Cook, DEA Models for Supply Chain Evaluation, Annals of Operations Research, 145, 35-49(2006)

Lima Junior, F.R. y L.C.R. Carpinetti, Quantitative models for supply chain performance evaluation: a literature review, Computers \& Industrial Engineering, 113(333), 346 (2017)

Navarro, K.P.S., Modelo De La Cadena De Abastecimiento Del Sector Madera y Muebles de La Región Caribe De Colombia, Entre Ciencia e Ingeniería, (14), 38-49(2013)

Nuñez-Pérez, N., M. Ortiz-Barrios, S. McClean, K. Salas-Navarro y otros dos autores, A Discrete-event simulation to reduce waiting time in accident and emergency departments: a case study in a district general clinic, International Conference on Ubiquitous Computing and Ambient Intelligence, 352-363 (2017)

Olivera, H.A.P. y B.I. V. Toro, Análisis Competitivo Del Sector Madera y Muebles De La Ciudad De Barranquilla, INGE CUC, 6(1), 195-213 (2010)

Ospina-Mateus, H., J. Acevedo-Chedid y otros tres autores, Model of Optimization of Mining Complex for the Planning of Flow of Quarry Production of Limestone in Multiple Products and with Elements for the Analysis of the Capacity, Workshop on Engineering Applications, 544-555 (2017)

Ovalle, A.M., O.L. Ocampo y M.T. Acevedo, Identificación De Brechas Tecnológicas En Automatización Industrial De Las Empresas Del Sector Metalmecánico De Caldas, Colombia, Ingeniería y Competitividad, 15(1) (2013)

Peñafiel, V., Cómo Desarrollar un Plan Estratégico de Logística para una Empresa, Logística Empresarial (2016)

Posada, J.G. A., Estudio de las Mejores Prácticas en Manufactura conocidas como herramientas de Producción Aplicadas en el Sector Metalmecánico de la ciudad de Medellín, Revista Universidad EAFIT, 40(133), 106-119 (2004)

Revista Dinero, Tecnoglass, como un vidrio templado, 1-2 (2015)

Rosenzweig, E.D., A.V. Rothy, J.W. Dean Jr, The Influence Of an Integration Strategy On Competitive Capabilities And Business Performance: An Exploratory Study Of Consumer Products Manufacturers, Journal of operations management, 21(4), 437-456 (2003)

Salas Navarro, K., Análisis de la Cadena de Abastecimiento del Sector Madera y Muebles de la ciudad de Barranquilla, Scientia et Technica, 16(49) (2011)

Salas Navarro, K., J.A. Chedid, N.M. Caruso y S.S. Sana, An Inventory Model Of Three-Layer Supply Chain Of Wood And Furniture Industry In The Caribbean Region Of Colombia, International Journal of Systems Science: Operations and Logistics, 5(1), 69-86 (2018)

Salas-Navarro, K., H. Maiguel-Mejia, J. Acevedo-Chedid, Metodología de Gestión de Inventarios para determinar los niveles de integración y colaboración en una cadena de suministro, Ingeniare. Revista Chilena de Ingeniería, 25(2), 326$337(2017)$

Stock, J.R. y D.M. Lambert, Strategic Logistics Management, Boston, McGraw-Hill Irwin (2007)

Swaminathan, J., S. Smith y N. Sadeh, Modeling Supply Chain Dynamics: A Multiagent Approach, Decision Sciences, 29(3), 607 (1998)

Trujillo Lora, J. C. y W. Iglesias Pinedo, Determinantes Del Crecimiento De Las Micro, Pequeñas Y Medianas Empresas Colombianas: El Caso Del Sector Metalmecánico, Semestre Económico, 15(32) (2012)

Viana, M., An Evaluation Of Supply Chain Management In A Global Perspective, Independent Journal of Management y Production, 6(1), 211 (2015) 